\title{
Universal concentration dependence of the Soret coefficient in aqueous systems
}

\author{
Simone Wiegand ${ }^{1}$, Hui Ning ${ }^{1}$ and Rio Kita ${ }^{2}$ \\ ${ }^{1}$ Research center Juelich GmbH, IFF - Soft Matter, \\ D-52428 Juelich, Germany \\ ${ }^{2}$ Department of Physics, Tokai University, Hiratsuka 259-1292, Japan
}

(Dated: May 8, 2007)

\begin{abstract}
We present measurements of aqueous low molecular weight mixtures and aqueous macromolecular solutions. The Soret coefficient $S_{\mathrm{T}}$ for ethanol, acetone and DMSO in water has been measured by an optical grating technique and all systems show a sign change around a molar fraction of water $x_{\text {water }}=0.85 \pm 0.05 . S_{\mathrm{T}}$ for poly(ethylene oxide), poly(N-isopropylacrylamide) and boehmite rods in ethanol/water mixtures shows also a sign change close to the same concentration. The occurring sign change concentration in the systems will be related to structural reorganisations in the solvent mixture and the Hildebrandt solubility parameter. We will also compare the experimental data within lattice calculations and simulations, which indicate that strong cross interactions are important for a sign change of the Soret coefficient.
\end{abstract}

\section{Introduction}

Aqueous mixtures and polymer solutions are used extensively for industrial purposes. Also for environmental reasons organic solvents are often replaced by water. The thermal diffusion behavior of aqueous mixtures is quite complex and mainly dominated by specific interactions. Some of the solute particles are even charged so that the situation is additionally complicated by long range interactions.

Often the thermal diffusion behavior of aqueous systems is complicated by sign changes. This is on the other hand a good chance to check the applied theories, whether they are capable to predict the observed sign changes correctly. For some other associating systems, already Prigogine et al. [1] explained qualitatively a sign change of $S_{\mathrm{T}}$ in the alcoholic mixtures methanol/benzene, ethanol/cyclohexane and hexanol/cyclohexane using a free energy concept. They found that a model based on potential energies of nearest-neighbor interactions alone led 
to contradictions when applied to alcohol mixtures, where the Soret coefficient changes sign. They emphasized that it is not sufficient only to discuss energetic contributions and argued that also the entropic contributions need to be considered. By assuming that alcohol molecules form complexes whose disruption results in a loss of local energy and a gain in local entropy, they were able to explain qualitatively the sign change of the Soret coefficient for these systems.

Recently, Soret coefficients of aqueous solutions have been calculated by molecular dynamics simulations $[2,3]$. The obtained data for ethanol/water and methanol/water showed quantitative agreement with experimental data. For the two other systems acetone/water and dimethylsulfoxide (DMSO)/water a sign change of the Soret coefficient was also predicted. The subsequent theoretical analysis in a lattice simulation revealed that large specific interactions are required to show a sign change in the thermophoretic behavior. In this lattice model cross interactions had to be larger than the interactions between the pure compounds in order to observe a sign change. A similar energetic argument was also derived from a two-chamber lattice calculation [4] for ethanol/water mixtures and by simulations of Lennard-Jones fluids [5]. Contrary to the alcohol mixtures examined by Prigogine $[1,6]$ for aqueous systems the energetic argumentation is quite successful.

There have been numerous experimental studies of water soluble polymers. In 1977, Giglio and Vendramini found a negative Soret coefficient for poly(vinyl alcohol) in water [7]. Recently, we investigated poly(ethylene oxide) (PEO) in ethanol/water mixtures $[8,9]$. It turned out that the observed sign change of the Soret coefficient of PEO in the solvent mixture at a water weight fraction of $x_{\text {water }}=0.92$ corresponded with the breakdown of the hydrogen bond network formed in pure water by addition of ethanol. In those studies the polymer concentration was so low $(1 \mathrm{~g} / \mathrm{L}$ and $5 \mathrm{~g} / \mathrm{L})$ that the observed sign change was caused by structural reorganisation in the vicinity of polymer and not due to interactions between the polymers. A sign change of the Soret coefficient was also observed for a diluted solution of poly(N-isopropylacrylamide) (PNiPAM) in ethanol [10]. The obtained Soret coefficient of PNiPAM was positive for lower temperatures $\left(\mathrm{T}<34^{\circ} \mathrm{C}\right)$, while showed a negative value for higher temperatures $\left(\mathrm{T}>34^{\circ} \mathrm{C}\right)$. This implies PNiPAM molecules move to the cold side for lower temperatures, whereas they move to the warm side for higher temperatures. In the investigated concentration range the transition temperature remained constant. In contrast the Soret coefficient of PNiPAM in water is always positive although the system undergoes a coil-globule transition indicating a drastic change of the solvent quality conditions. Piazza and coworkers found for numerous aqueous macromolecular and colloidal suspensions a universal characteristic dependence on 


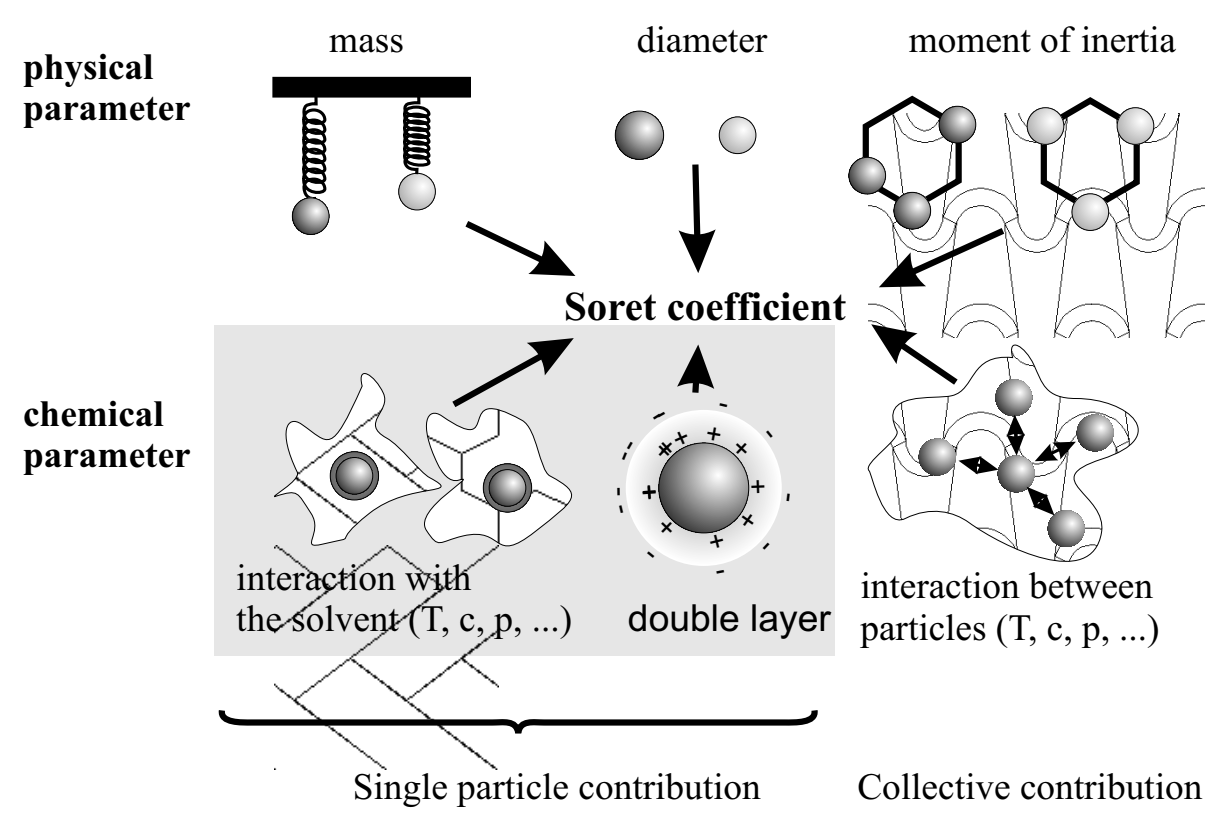

Figure 1: Main features determining the Soret coefficient

temperature [11].

The main features that control thermal diffusion behavior are displayed in Figure 1. The physical parameters are easily accessible particle properties such as mass, moment of inertia and size of the considered molecule or particle. The moment of inertia is closely related to the flexibility of the molecule and is only constant for small rigid molecules, where it might be used as parameter characterizing the system [12]. The chemical contributions can be roughly divided in single particle and collective contributions. Single particle contributions such as the formation of an ionic double layer or the break-down of hydrogen bond network in the surrounding solvent will dominate the behavior in the highly diluted regime. Measurements indicate that the first shell of the surrounding solvent molecules, in particular, is important [13]. In concentrated solutions the interactions between solute molecules also need to be considered. Additionally, it should be mentioned that for low-molecular weight molecules kinetic effects are important, while for high molecular weight substances they can be neglected.

In the present paper we want to discuss the thermal diffusion behavior of aqueous low molecular weight mixtures as ethanol/water, acetone/water and DMSO/water. The results are compared with recent simulations. Further, we discuss polymer solutions of PEO and PNiPAM in ethanol/water mixtures and rod like colloidal particles in the same solvent mixture. For both systems the sign change concentration is correlated with the solvation properties of the solution. 


\section{Experimental}

\subsection{Sample preparation and refractive index increments}

The weight- and number- averaged molecular weight of PEO are $M_{w}=2.65 \times 10^{5} \mathrm{~g} / \mathrm{mol}$ and $M_{n}=2.36 \times 10^{5} \mathrm{~g} / \mathrm{mol}$, respectively, determined by GPC according to a poly(styrene) calibration. A dispersion of boehmite needles in demineralised water was prepared according to Buining [14]. Transmission electron microscopy yielded an average length of $251 \mathrm{~nm}$ (polydispersity: $40 \%$ ), and an average thickness of $8 \mathrm{~nm}$. A detailed description of the sample preparation of PEO and boehmite is given in Ref. [15]. PNiPAM was purified by a fractionation method [10]. One fraction was used in this study which had the weight-averaged molecular weight $M_{w}=3.0 \times 10^{6} \mathrm{~g} / \mathrm{mol}$ with a polydispersity $M_{w} / M_{n}=1.20$. Details of sample preparation and characterizations are described elsewhere [10]. In this study $1.0 \mathrm{~g} / \mathrm{L} \mathrm{PNiPAM}$ in water was prepared [9]. The solvents acetone (Laborchemie Handels-GmbH, purity> 99.9\%), DMSO (Sigma-Aldrich,purity $\geq 99.9 \%$ ) and absolute ethanol (Riedel-de Haën, HPLC-grade) were used without further purification. Further details can be found elsewhere [16]. To all aqueous solutions we added a tiny amount (roughly $10^{-5} \mathrm{wt}$ ) of the dye basantol yellow [17], which was dissolved in the solvents. Typically the optical density was adjusted to $2-3 \mathrm{~cm}^{-1}$ at a wavelength of $\lambda=488 \mathrm{~nm}$. The temperature was controlled by a circulating water bath and temperature fluctuations were always smaller than $\Delta T= \pm 0.02 \mathrm{~K}$. The refractive index increments with temperature and concentration were determined with a Michelson interferometer [18].

\subsection{TDFRS set-up and data analysis}

The experimental setup comprises a standard holographic grating setup with an argon ion laser for writing and a helium-neon laser for reading out the grating operating at a wavelength of $\lambda_{\mathrm{w}}=488 \mathrm{~nm}$ and $\lambda_{\mathrm{r}}=633 \mathrm{~nm}$, respectively. The polarized beam of the argon ion laser is split into two beams of equal intensity, which are brought to interference within the sample in the cell under a variable angle. By varying the angle the grating vector and the fringe spacing can be changed. A typical grating vector in the experiments was around $q=3100 \mathrm{~cm}^{-1}$, which corresponds to a fringe spacing around $d=20 \mu \mathrm{m}$. The added dye with a strong absorption band at $\lambda=488 \mathrm{~nm}$ converts the optical grating in a temperature grating. The obtained normalized heterodyne signal is related to the Soret coefficient $S_{\mathrm{T}}$ and diffusion coefficient $D$. Further details about the set-up and the analysis can be found elsewhere. [17] 


\section{Results and discussion}

\subsection{Aqueous solvent mixtures}

We performed thermal diffusion measurements for ethanol, acetone and DMSO in water. In Fig.2A-C the Soret coefficients $S_{\mathrm{T}}$ for all three systems are shown as function of the molar fraction of organic solvent $x_{\text {solvent. }}$ In the water-rich region the Soret coefficient of acetone $S_{\mathrm{T}}$ decreases with increasing acetone concentration, reaches a minimum at a molar fraction of $x_{\text {acetone }}=0.5$ and again increases for acetone-rich solutions. The Soret coefficients of DMSO and ethanol are quite similar. In the water-rich regime $S_{\mathrm{T}}$ decreases with decreasing water content and reaches almost a plateau or wide minimum for $x_{\text {water }}<0.6$. All three systems show a sign change of Soret coefficient with concentration in the water rich region for acetone, DMSO and ethanol at $x_{\text {acetone }}=0.11, x_{\text {DMSO }}=0.2$ and $x_{\text {ethanol }}=0.14$, respectively. For ethanol $(\mathrm{cp}$. Fig 2C) we compare the experimental data with a lattice calculation [4], which predicts a sign change for a much higher water content and overshoots the experimental data in the water rich and water poor regime. The sign change in this lattice calculation was only found, when the cross interactions are stronger than the like interactions. Our experimental data confirm the sign change predicted by non-equilibrium molecular dynamic (NEMD) for acetone and DMSO in water. The simulation data agree reasonably well with the experimental results, although the simulations do not predict the pronounced minimum for acetone and overshot the experimental values at low DMSO concentrations. In the NEMD simulations the cross interactions were computed by Lorentz-Berthelot rules or by geometric average [19], which reproduced the sign change in the aqueous mixtures. In contrast in lattice calculations [4], lattice simulations [3] and simulations of Lennard Jones fluids [5] the mixed interactions need to be larger than the pure interactions in order to observe a sign change with concentration. The reason for the different behavior is probably that the NEMD are dominated by the specific interactions as hydrogen bond formation and not by the Lennard-Jones interactions.

In Fig.2D we plotted the Soret coefficient normalized by the minimal values versus the molar fraction of solvent. We added also literature data [20] for the system methanol/water, which changes sign at methanol content of $x_{\text {methanol }}=0.15$. Over a wide concentration range the normalized data overlap. The system specific characteristics are especially displayed in the water rich regime. Here obviously the different hydrogen bond capability leads to different sign change concentrations. We would like to point out that the concentration where the Soret coefficient changes sign is correlated with the concentration where the hydrogen bond network 


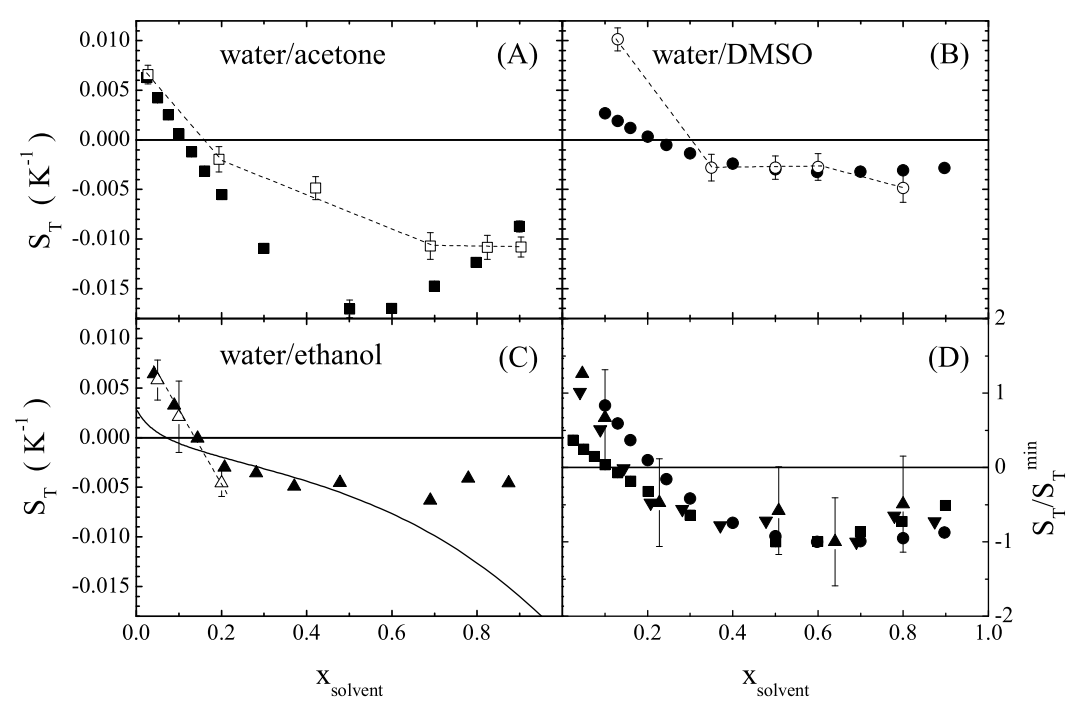

Figure 2: (A)-(C) A comparison of experimental (solid symbols) and simulation (open symbols) data for Soret coefficient of ethanol $(\boldsymbol{\Delta})$, acetone $(\boldsymbol{\square})$ and DMSO $(\bullet)$ in water as function of the molar fraction of organic solvent. The dashed lines are guides to the eye. The solid line in (C) refers to a lattice calculation by Luettmer-Strathmann [4]. (D) This part shows the Soret coefficients normalized by the minimal value for each system. Literature data for methanol have been added $(\boldsymbol{\nabla})[20]$.

of pure water breaks down by addition of second component. According to NMR studies the concentrations are $x_{\text {acetone }}^{\text {hyd }}=0.06[21], x_{\mathrm{DMSO}}^{\text {hyd }}=0.10[22]$ and $x_{\text {ethanol }}^{\text {hyd }}=0.08$ [23], respectively. A direct correlation of the thermal diffusion properties with one of the Hildebrandt solubility parameters works only to some extend [16]. Typically, either the system acetone or DMSO did not fit in the series build up by ethanol/water and methanol/water.

\subsection{Polymers and colloids in ethanol/water mixtures}

Fig. 3 shows the Soret coefficient of PEO, PNiPAM and boehmite rods in ethanol/water as a function of ethanol molar fraction at room temperature. Our experimental studies give some insight into conditions that lead to unusual thermal diffusion in polymer solutions [8]. It turns out that the solvent quality is very important. Positive Soret coefficients are observed for all polymer systems in pure water, which is an excellent solvent, while negative Soret coefficients are observed for low water content of the solution that is under poorer solvent conditions. 


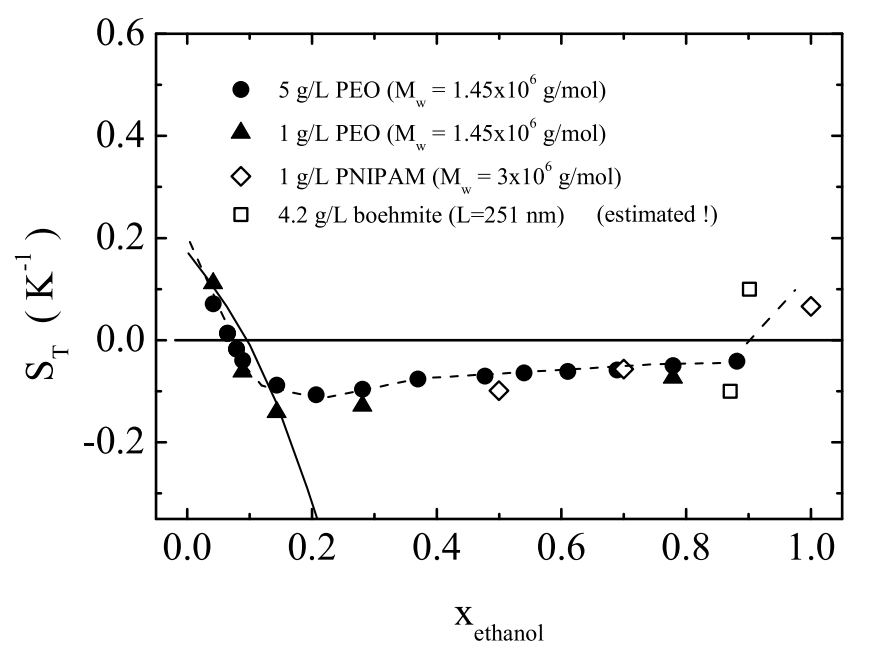

Figure 3: Soret coefficient $S_{\mathrm{T}}$ for PEO, PNiPAM and boehmite rods [15,24] in ethanol/water mixtures at room temperature versus the molar fraction of ethanol. The solid line refers to a lattice calculation. [9] The symbols indicate the systems listed in the legend and the dotted line is a guide to the eye.

This observations is in agreement with a lattice calculation [25] and a recent simulation for a polymer represented by a generic bead-spring model [5]. Both studies showed that a better solvent quality causes a higher affinity for polymers to the cold side. Also the negative Soret coefficient reported [7] for poly(vinyl alcohol) in water at $25^{\circ} \mathrm{C}$ agrees with our findings since the system is very close to poor solvent conditions [26]. In the case of pure ethanol we observed for PNiPAM and boehmite, another sign change to a positive Soret coefficient. Whether PEO changes also the sign in pure ethanol could not been measured, because only very low molecular weight oligomers $(M<2000 \mathrm{~g} / \mathrm{mol})$ can be dissolved. And for those oligomers the concentration signal was too weak to be analyzed. Comparing the three different systems one finds the same trend for all three systems, indicating that the structural reorganisation in the mixed solvent leads to a sign change of the Soret coefficient of the solute.

Our investigation leads us to expect other polymer systems to yield changes in sign of polymer Soret coefficients. For example, a solution of a copolymer in a single solvent may change sign as a function of composition of the copolymer, if the chains are composed of two types of segments, one with highly attractive and one with net repulsive segment-solvent interactions. 


\section{Conclusion}

In this study we have seen, that the concentration dependence of aqueous low molecular weight solutions and complex mixtures is quite similar. In all mixtures the sign change of the Soret coefficients seem to be close to the concentration, where the hydrogen bond network of pure water breaks by adding a second component. All investigated complex solutions were diluted, so that the thermal diffusion behavior was mainly determined by single particle contributions such as the interface of the solute particle and the surrounding solvent. For the low molecular weight mixtures acetone/water and DMSO/water we could confirm the sign change by NEMD predicted. The agreement between experimental results and simulation data is reasonable in the entire concentration range. The lattice calculations for ethanol/water model could only predict the sign change. A better quantitative agreement is only possible after a refinement of the model.

\section{Acknowledgments}

The authors would like to thank Florian Müller-Plathe and Bernard Rousseau for fruitful discussions. We thank Malte Kleemeier for carefully reading the manuscript. This work was partially supported by the Deutsche Forschungsgemeinschaft grant Wi 1684 .

\section{References}

[1] I. Prigogine, L. Debrouckere, and R. Amand. Recherches sur la thermodiffusion en phase liquide 2. Physica, 16 (1950), 851-860.

[2] B. Rousseau, C. Nieto-Draghi, and J. Bonet Avalos. The role of molecular interaction in the change of sign of the Soret coefficient. Europhys. Lett., 67 (2004), 976-982.

[3] C. Nieto-Draghi, J. B. Avalos, and B. Rousseau. Computing the Soret coefficient in aqueous mixtures using boundary driven nonequilibrium molecular dynamics. J. Chem. Phys., 122 (2005), art. no. -114503 .

[4] J. Luettmer-Strathmann. Lattice model for thermodiffusion in polymer solutions. Int. J. Thermophys., 26 (2005), 1693-1707. 
[5] M. M. Zhang and F. Müller-Plathe. The Soret effect in dilute polymer solutions: influence of chain length, chain stiffness and solvent quality. J. Chem. Phys., 125 (2006), art. no.124903.

[6] I. Prigogine, L. Debrouckere, and R. Amand. Recherches sur la thermodiffusion en phase liquide .1. Physica, 16 (1950), 577-598.

[7] M. Giglio and A. Vendramini. Soret-type motion of macromolecules in solution. Phys. Rev. Lett., 38 (1977), 26-30.

[8] B.-J. de Gans, R. Kita, S. Wiegand, and J. Luettmer Strathmann. Unusual thermal diffusion in polymer solutions. Phys. Rev. Lett., 91 (2003), 245501.

[9] R. Kita, S. Wiegand, and J. Luettmer-Strathmann. Sign change of the Soret coefficient of poly(ethylene oxide) in water/ethanol mixtures observed by TDFRS. J. Chem. Phys., 121 (2004), 3874-3885.

[10] R. Kita, G. Kircher, and S. Wiegand. Thermally induced sign change of Soret coefficient for dilute and semidilute solutions of poly. n-isopropylacrylamide. in ethanol. J. Chem. Phys., 121 (2004), 9140-9145.

[11] S. Iacopini, R. Rusconi, and R. Piazza. The "macromolecular tourist": Universal temperature dependence of thermal diffusion in aqueous colloidal suspensions. Eur. Phys. J. E, 19 (2006), 59-67.

[12] C. Debuschewitz and W. Köhler. Molecular origin of thermal diffusion in benzene plus cyclohexane mixtures. Phys. Rev. Lett., 87 (2001), art. no.-055901.

[13] S. Wiegand. Thermal diffusion in liquid mixtures and polymer solutions. J.Phys.:Condens. Matter, 16 (2004), R357-R379.

[14] P. A. Buining, C. Pathmamanoharan, J. B. H. Jansen, and H. N. W. Lekkerkerker. Preparation of colloidal boehmite needles by hydrothermal treatment of aluminum alkoxide precursors. J. Am. Ceram. Soc., 74 (1991), 1303-1307.

[15] B. J. de Gans, R. Kita, B. Müller, and S. Wiegand. Negative thermodiffusion of polymers and colloids in solvent mixtures. J. Chem. Phys., 118 (2003), 8073-8081. 
[16] H. Ning and S. Wiegand. Experimental investigation of the Soret effect in acetone/water and water/dimethylsulfoxide mixtures. J. Chem. Phys., 125 (2006), art. no.-221102.

[17] H. Ning, R. Kita, H. Kriegs, J. Luettmer-Strathmann, and S. Wiegand. Thermal diffusion behavior of nonionic surfactants in water. J. Phys. Chem. B, 110 (2006), 10746-10756.

[18] A. Becker, W. Köhler, and B. Müller. A scanning michelson interferometer for the measurement of the concentration and temperature derivative of the refractive- index of liquids. Ber. Bunsen-Ges. Phys. Chem., 99 (1995), 600-608.

[19] C. Nieto-Draghi. Transport and structural properties of aqueous solutions of organic solvents. PhD thesis, Universitat Rovira i Virgili, (2003).

[20] L. J. Tichacek, W. S. Kmak, and H. G. Drickamer. Thermal diffusion in liquids - the effect of non-ideality and association. J. Phys. Chem., 60 (1956), 660-665.

[21] K. Mizuno, T. Ochi, and Y. Shindo. Hydrophobic gydration of acetone probed by nuclear magnetic resonance and infrared: Evidence for the interaction $\mathrm{C}-\mathrm{H} \ldots \mathrm{OH}_{2}$. J. Chem. Phys., 109 (1998), 9502-9507.

[22] K. Mizuno, S. Imafuji, T. Ochi, and S. Maeda. Hydration of the CH groups dimethyl sulfoxide probed by NMR and IR. J. Phys. Chem. B, 104 (2000), 11001-11005.

[23] A. Coccia, P. L. Indovina, F. Podo, and V. Viti. PMR studies on structures of water-ethyl alcohol mixtures. Chem. Phys., 7 (1975), 30-40.

[24] A quantitative determination of the Soret coefficient was not possible due to preferential adsorption of the dye at the surface of the colloidal particles, which lead to an inhomogeneous heating within the colloidal suspension.

[25] J. Luettmer-Strathmann. Two-chamber lattice model for thermodiffusion in polymer solutions. J. Chem. Phys., 119 (2003), 2892-2902.

[26] D. Eagland and N. J. Crowther. Hydrophobic interactions in dilute-solutions of polyvinylalcohol). Faraday Symp. Chem. Soc., 17 (1982), 141-160. 